\title{
Ischemic Vaso-occlusive Retinopathy as Initial Presentation in Pediatric Systemic Lupus Erythematosus: A Case Report
}

\author{
Jin Hwan Jeon, M.D., Hye Yeon Choi, M.D., Jung Woo Rhim, M.D., Ph.D., Soo Young Lee, M.D., Ph.D., \\ Young Gun Park, M.D., Ph.D., Dae Chul Jeong, M.D., Ph.D. \\ Department of Pediatrics, College of Medicine, The Catholic University of Korea, Seoul, Korea
}

\begin{abstract}
Ischemic vaso-occlusive retinopathy as an initial manifestation is rare in pediatric systemic lupus erythematosus (pSLE). A 13-year-old girl presented with two months' history of papules and crusts with fatigue, weight loss, and abrupt hair loss. Pancytopenia and findings compatible with SLE, including positive direct Coombs' test, antinuclear antibody (Ab), anti-double stranded DNA Ab, anti-Smith Ab, anti-ribonucleoprotein Ab, lupus anticoagulant, anti- $\beta 2$ glycoprotein Immunoglobulin $\mathrm{G}$, and anti-cardiolipin $\mathrm{Ab}$, were detected. Bi-nasal hemianopsia was detected. Initial visual acuity was hand motion in the right eye and 15/20 in the left. Fundoscopy showed massive exudation around the optic disc with macular edema, vascular sheathing with perivascular hemorrhage in the whole retina, and ghost vessels in the peripheral retina. Intravitreal triamcinolone injection and dexamethasone implant injection were administered. Visual symptoms improved but did not recover. Methylprednisolone therapy and photocoagulation improved visual acuity and fever. Early intervention for retinopathy in pSLE can help prevent vision-loss. (J Rheum Dis 2022;29:52-55)
\end{abstract}

Key Words. Lupus erythematosus, systemic, Pediatrics, Retinal diseases

\section{INTRODUCTION}

Pediatric systemic lupus erythematosus (pSLE) is a chronic autoimmune inflammatory disease involving multiple organs, with a prevalence of 3.3 24 per 100,000 people, and its clinical course shows more severe pattern than adults [1]. The clinical manifestation of PSLE is dependent upon multiple organs, characterized by inflammation due to autoimmunity and immune dysfunction $[1,2]$.

Abnormal ocular findings are detected in one-third of SLE patients and may be present at the initial manifestations or appear during the clinical course $[3,4]$. These ophthalmologic manifestations can be clinically silent for several months and are correlated with disease activity. Keratoconjunctivitis sicca is the most common ocular finding, while retinal and choroidal involvement is asso- ciated with vision loss $[3,5]$. Ocular involvement in PSLE is rare and shows a more aggressive phenotype with worse outcome than adults [6,7].

The most serious ophthalmologic presentation in SLE is retinopathy accompanied by micro-vessel disease, which is also characterized by a cotton wool patch with or without intraretinal hemorrhage [3-5]. Retinopathy is very rare among pSLE patients, however its incidence has not been published [8]. Moreover, some patients might exhibit severe vaso-occlusive retinopathy, which progresses to irreversible blindness in pSLE [9]. Therefore, the initial detection of ocular manifestations in pSLE may be important, because vision loss may develop despite aggressive treatment.

Here, we report a case of vaso-occlusive lupus retinopathy as an initial manifestation of pSLE.

This study was approved by the Institutional Review

Received : May 10, 2021, Revised : June 1, 2021, Accepted : June 12, 2021

Corresponding to : Dae Chul Jeong (iD http://orcid.org/0000-0003-0934-817X

Department of Pediatrics, Seoul St. Mary's Hospital, College of Medicine, The Catholic University of Korea, 222 Banpo-daero, Seocho-gu, Seoul 06591, Korea. E-mail : dcjeong@catholic.ac.kr 
Board of Seoul St. Mary's Hospital (KC21ZAS10288). Informed consent was waived by the board.

\section{CASE REPORT}

A 13-year-old girl was admitted with dizziness for a month. She had a two-month history of papules and crusts on the face and extremities with fatigue, weight loss, and abrupt hair loss. The patient had no significant medical history or a specific family history.

She had not presented fever before hospitalized, her body temperature was $38.3^{\circ} \mathrm{C}$ on admission. She complained of myodesopsia that binasal hemianopsia was observed on eye examination. Blood tests revealed pancytopenia with a white blood cell count of $2,260 / \mu \mathrm{L}$, hemoglobin of $7.0 \mathrm{~g} / \mathrm{dL}$, and platelet count of $93 \times 103 / \mu \mathrm{L}$. C3 level was $60 \mathrm{mg} / \mathrm{dL}(86 \sim 182 \mathrm{mg} / \mathrm{dL})$. The baseline study showed positive direct Coombs' test and several autoantibodies including antinuclear antibody $(\mathrm{Ab})(1: 2,560$, speckled), anti-double stranded DNA Ab $(52.8 \mathrm{IU} / \mathrm{mL}$, normally less than $27 \mathrm{IU} / \mathrm{mL}$ ), anti-Smith $\mathrm{Ab}$ (strong positive), anti-ribonucleoprotein $\mathrm{Ab}$ (153.5 U, normally less than $20 \mathrm{U}$ ), perinuclear anti-neutrophil cytoplasmic $\mathrm{Ab}$ (p-ANCA, immunofluorescence assay), lupus anticoagulant (LA) (50.5 seconds, normally less than 38 seconds), anti $\beta$ 2-glycoprotein 1 immunoglobulin (Ig) G (86.1 CU, normally less than $20 \mathrm{CU}$ ), and Clq immune complex (5.05, normally less than $3.2 \mu \mathrm{g} / \mathrm{mL}$ ). Rheumatoid factor, anti-Lo/SSA Ab, anti-Ra/SSB Ab, anti-Jo- $1 \mathrm{Ab}$, anti-centromere $\mathrm{Ab}$, anti-cardiolipin $\mathrm{IgG} / \mathrm{IgM}$, anti-myeloperoxidase $\mathrm{Ab}$, and anti-proteinase 3-antineutrophil cytoplasmic Ab (PR3-ANCA) were negative. Clinical symptoms and laboratory findings were compatible with SLE according to the Systemic Lupus International Collaborating Clinics criteria.

On ophthalmic examination, visual acuity was hand- motion in the right eye and 15/20 in the left eye. The eyelid, conjunctiva, cornea, and iris were found to be normal. Fundus examination revealed exudate at the optic disc rim, mildly edematous macula, and vascular sheathing around retinal vessels (Figure 1); perivascular hemorrhage was found around the ghost vessels in the whole retina of both eyes (Figure 2). Magnetic resonance imaging of the orbit showed no abnormal findings in the optic nerves or brain.

On the third day after admission, the patient presented with sustained fever, and pancytopenia persisted on follow-up laboratory data. She was treated with oral hydroxychloroquine with high-dose intravenous methylprednisolone of $20 \mathrm{mg} / \mathrm{kg} /$ day $(1 \mathrm{~g} / \mathrm{kg} /$ day $)$ for 3 days as a systemic treatment. Triamcinolone was injected into the right vitreous body for four days, and dexamethasone intravitreal implant (Ozurdex; Allergan Plc, Dublin, Ireland) was inserted into the left vitreous body.

Her symptoms did not improve significantly after the initial treatment, and she was again treated with additional methylprednisolone therapy. Panretinal photocoagulation was performed on hospital days 9 and 11 in order to prevent vessel neogenesis in the ischemic retina.

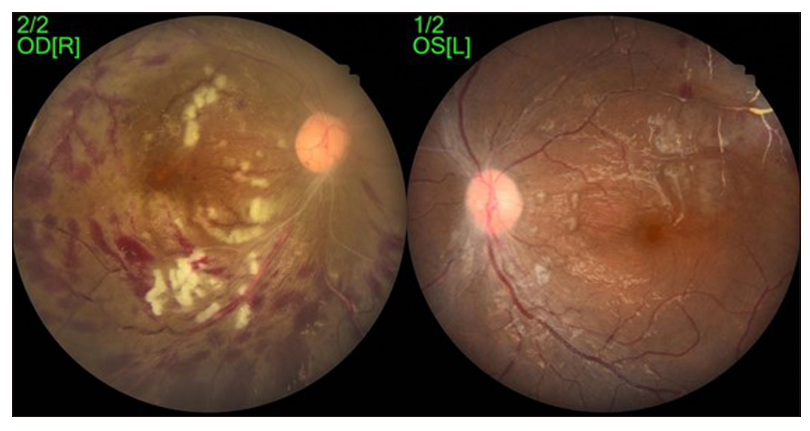

Figure 2. Fundoscopic findings showed vascular sheathing with perivascular hemorrhage at whole retina and ghost vessels at periphery retina at both eyes.
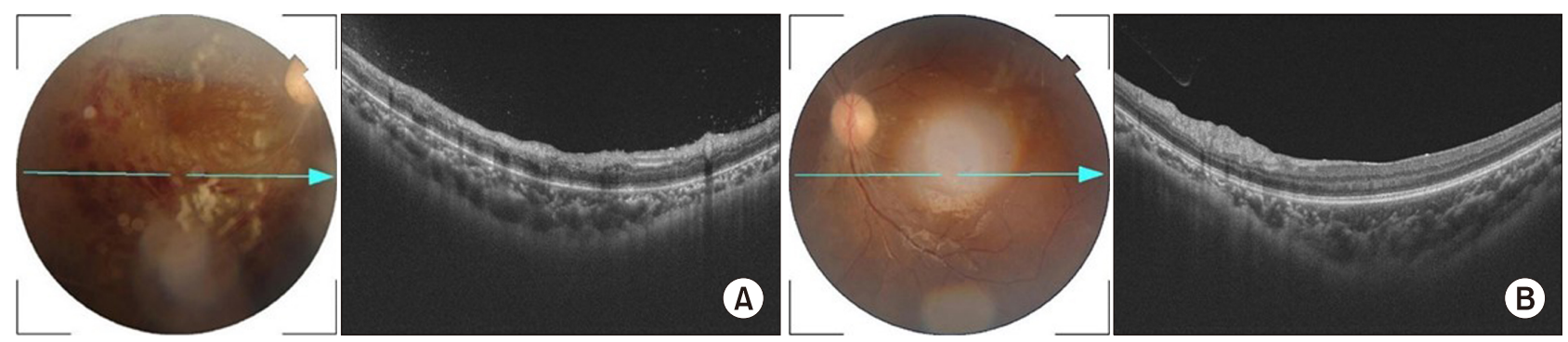

Figure 1. Macular optical coherence tomographs. Massive exudation at both eyes with macular edema was noted. Right eye (A), left eye (B). 
After treatment, the patient's clinical manifestations and visual symptoms improved, confirmed by laboratory findings. The patient was discharged with a plan of further follow-up in the future.

\section{DISCUSSION}

Although the prevalence varies considerably between studies, approximately one-third of SLE patients are reported to have ocular complications [3]. Occlusive retinopathy, which is a severe form of ocular complication, is known to have a prevalence of $3 \% \sim 11 \%$ among the adult population and is reported to be rarer in the pediatric population $[8,10]$. Due to the low prevalence in children, many pediatricians do not pay attention to the ocular involvement in PSLE patients.

As shown in previous studies, the occurrence of retinopathy as the initial symptom of SLE is rare. From a retrospective case control study in Chinese adult SLE patients, ophthalmic complications can occur at any stage of disease progression, with a median duration of 12 months [11]. Several adult patients with retinopathy have been reported in South Korea as well $[12,13]$.

Ocular complications and visual defects have been reported in several pSLE patients worldwide. According to a study in Brazil, two patients showed blindness after the diagnosis of pSLE [9]. One of them had photophobia, pain, and impairment of visual acuity one month after the initial diagnosis with systemic methylprednisolone treatment. Another patient suffered from blurry vision and photophobia 13 years after the diagnosis. In another case report, two pSLE patients developed acute unilateral vision changes after the diagnosis [8]. Patients who are diagnosed and treated early have superior visual outcome. In a recent study in China, a patient was checked by an ophthalmologist and no abnormal findings were found at the time of the initial diagnosis of pSLE [14]. However, the patient complained of sudden vision loss in the left eye on the fourth day of hospitalization and was diagnosed with retinopathy.

The visual prognosis of retinal involvement depends on the pattern of retinopathy. Some patients develop massive obstruction of retinal arterioles or artery branches, causing ischemic lupus retinopathy with retinal ischemia and angiogenesis, usually leading to poor visual outcome [5]. According to a study by Jabs et al., about $50 \%$ of SLE patients with vaso-occlusive retinopathy have a vision worse than 20/200 [15]. Thus, patients suspected of hav- ing an ophthalmologic complication require immediate proper ophthalmologic evaluation and intervention.

The association between antiphospholipid Abs, such as LA or anti-cardiolipin Abs, and lupus retinopathy has been proven by numerous studies. In Italy, 21.3\% $23.3 \%$ of SLE patients presented positive results for antiphospholipid antibodies [4]. Along with the vasculitis caused by SLE, the coagulating tendency of antiphospholipid antibodies explains the increased risk of vessel obstruction. In this patient, LA and anti- $\beta 2$ glycoprotein 1 IgG were positive, which might be related to vaso-occlusive retinopathy.

Ophthalmologic complications of SLE are closely related to disease activity in general [3]. Controlling systemic symptoms may be helpful in alleviating the ophthalmologic symptoms. Conventional medications for SLE include antimalarial drugs, immunosuppressants, and steroids. Systemic steroid therapy is required in patients with SLE accompanied by severe vaso-occlusive retinopathy. The goal of the SLE retinopathy treatment is to prevent angiogenesis and vitreous hemorrhage to improve visual outcome; panretinal photocoagulation and vitrectomy are useful in this process $[3,4]$.

In this case, the patient complained of myodesopsia and underwent ophthalmic evaluation at the time of diagnosis. Her ocular symptoms improved with combination therapy, including systemic and local treatment. As ocular involvement may remain clinically silent or show symptoms that are not too uncomfortable, all patients with SLE should undergo an ophthalmologic evaluation at an early phase of the disease progression, regardless of the presence of symptoms, to prevent vision-loss.

\section{SUMMARY}

We report a case of a pediatric SLE patient with vaso-occlusive retinopathy, which is a rare symptom, at the time of diagnosis. Therefore, we recommend that every patient must undergo ophthalmic evaluation regardless of the ocular manifestation.

\section{CONFLICT OF INTEREST}

No potential conflict of interest relevant to this article was reported. 


\section{AUTHOR CONTRIBUTIONS}

Conception or design: D.C.J., J.H.J., and H.Y.C. Acquisition, analysis, or interpretation of data: J.H.J., J.W.R., S.Y.L., and Y.G.P. Drafting the work or revising: D.C.J., J.H.J., and H.Y.C. Final approval of the manuscript: D.C.J.

\section{REFERENCES}

1. Harry O, Yasin S, Brunner H. Childhood-onset systemic lupus erythematosus: a review and update. J Pediatr 2018; 196:22-30.e2.

2. Kaul A, Gordon C, Crow MK, Touma Z, Urowitz MB, van Vollenhoven R, et al. Systemic lupus erythematosus. Nat Rev Dis Primers 2016;2:16039.

3. Dammacco R. Systemic lupus erythematosus and ocular involvement: an overview. Clin Exp Med 2018;18:135-49.

4. Dammacco R, Procaccio P, Racanelli V, Vacca A, Dammacco F. Ocular involvement in systemic lupus erythematosus: the experience of two tertiary referral centers. Ocul Immunol Inflamm 2018;26:1154-65.

5. Silpa-archa S, Lee JJ, Foster CS. Ocular manifestations in systemic lupus erythematosus. Br J Ophthalmol 2016;100: 135-41.

6. Ambrose N, Morgan TA, Galloway J, Ionnoau Y, Beresford MW, Isenberg DA; UK JSLE Study Group. Differences in disease phenotype and severity in SLE across age groups. Lupus 2016;25:1542-50.
7. Watson L, Leone V, Pilkington C, Tullus K, Rangaraj S, McDonagh JE, et al. Disease activity, severity, and damage in the UK Juvenile-Onset Systemic Lupus Erythematosus Cohort. Arthritis Rheum 2012;64:2356-65.

8. Donnithorne KJ, Read RW, Lowe R, Weiser P, Cron RQ, Beukelman T. Retinal vasculitis in two pediatric patients with systemic lupus erythematosus: a case report. Pediatr Rheumatol Online J 2013;11:25.

9. Almeida RT, Aikawa NE, Sallum AM, Jesus AA, Sa LC, Silva CA. Irreversible blindness in juvenile systemic lupus erythematosus. Lupus 2011;20:95-7.

10. Stafford-Brady FJ, Urowitz MB, Gladman DD, Easterbrook M. Lupus retinopathy. Patterns, associations, and prognosis. Arthritis Rheum 1988;31:1105-10.

11. Gao N, Li MT, Li YH, Zhang SH, Dai RP, Zhang SZ, et al. Retinal vasculopathy in patients with systemic lupus erythematosus. Lupus 2017;26:1182-9.

12. Song YH, Kim CG, Kim SD, Kim YY, Choe JY. Systemic lupus erythematosus presenting earlier as retinal vasoocclusion. Korean J Intern Med 2001;16:210-3.

13. Kang WH, Kim J, Choi JH, Kim MJ. Two cases of systemic lupus erythematosus presenting earlier as retinal microangiopathy. J Korean Rheum Assoc 2006;13:251-5.

14. Huang G, Shen H, Zhao J, Mao J. Severe vaso-occlusive lupus retinopathy in the early stage of a pediatric patient with systemic lupus erythematosus: a case report. Medicine (Baltimore) 2020;99:e19875.

15. Jabs DA, Fine SL, Hochberg MC, Newman SA, Heiner GG, Stevens MB. Severe retinal vaso-occlusive disease in systemic lupus erythematous. Arch Ophthalmol 1986;104: 558-63. 